\title{
AN INEQUALITY OF SCHUR AND AN INEQUALITY OF NEWTON
}

K. V. MENON

Let $E_{r}^{1}$ and $H_{r}^{1}$ denote the elementary and complete symmetric functions of the $r$ th order in $\alpha_{1}, \alpha_{2}, \cdots, \alpha_{m}$ respectively.

If

$$
E_{r}=E_{r}^{1} /\left(\begin{array}{l}
m \\
r
\end{array}\right)
$$

and

$$
H_{r}=H_{r}^{1} /\left(\begin{array}{c}
m+r-1 \\
r
\end{array}\right)
$$

then we have two well-known inequalities

$$
E_{r}^{2} \geqq E_{r-1} E_{r+1}
$$

and

$$
H_{r}^{2} \leqq H_{r-1} H_{r+1}
$$

The inequality (1.3) is known as Newton's inequality and is true when $\alpha_{1}, \alpha_{2}, \cdots, \alpha_{m}$ are real numbers. The inequality (1.4), given by Professor Schur, is true when $\alpha_{1}, \alpha_{2}, \cdots, \alpha_{m}$ are nonnegative reals. In [1], Whiteley has given the following generalizations of (1.3) and (1.4): Let

(1.5) $E_{r}^{1}(K)=\sum \lambda_{i_{1}} \lambda_{i_{2}} \cdots \lambda_{i_{m}} \alpha_{1}^{i_{1}} \alpha_{2}^{i_{2}} \cdots \alpha_{m}^{i_{m}}, \quad i_{1}+i_{2}+\cdots+i_{m}=r$,

$$
\lambda_{i}=\left(\begin{array}{c}
K \\
i
\end{array}\right)
$$

and $K$ any positive real number. Similarly we define,

$$
\begin{aligned}
& H_{r}^{1}(K)=\sum \delta_{i_{1}} \delta_{i_{2}} \cdots \delta_{i_{m}} \alpha_{1}^{i_{1}} \alpha_{2}^{i_{2}} \cdots \alpha_{m}^{i_{m}} \\
& i_{1}+i_{2}+\cdots+i_{m}=r
\end{aligned}
$$

where

$$
\delta_{i}=(-1)^{i}\left(\begin{array}{c}
K \\
i
\end{array}\right)
$$

Received by the editors December 5, 1968. 
and $K$ any negative real number. In [1], it is proved that if

$$
\begin{aligned}
& E_{r}(K)=E_{r}^{1}(K) /\left(\begin{array}{c}
K m \\
r
\end{array}\right), \\
& H_{r}(K)=H_{r}^{1}(K) /\left(\begin{array}{c}
K m \\
r
\end{array}\right),
\end{aligned}
$$

then

$$
\begin{aligned}
& {\left[E_{r}(K)\right]^{2} \geqq E_{r-1}(K) \cdot E_{r+1}(K),} \\
& {\left[H_{r}(K)\right]^{2} \leqq H_{r-1}(K) \cdot H_{r+1}(K) .}
\end{aligned}
$$

In this paper we prove that (1.9) and (1.10) are true for a more general type of nonsymmetric functions

$$
\begin{aligned}
& E_{r}\left(K_{1}, K_{2}, \cdots, K_{m}\right) \\
& H_{r}\left(K_{1}, K_{2}, \cdots, K_{m}\right)
\end{aligned}
$$

which are defined in the following way: Let

$$
\begin{aligned}
& E_{r}^{1}\left(K_{1}, K_{2}, \cdots, K_{m}\right)= \sum\left(\begin{array}{c}
K_{1} \\
i_{1}
\end{array}\right)\left(\begin{array}{c}
K_{2} \\
i_{2}
\end{array}\right) \cdots\left(\begin{array}{c}
K_{m} \\
i_{m}
\end{array}\right) \alpha_{1}^{i_{1}} \alpha_{2}^{i_{2}} \cdots \alpha_{m}^{i_{m}}, \\
& i_{1}+i_{2}+\cdots+i_{m}=r ; \\
& H_{r}^{1}\left(K_{1}, K_{2}, \cdots, K_{m}\right)= \sum\left(\begin{array}{c}
K_{1}+i_{1}-1 \\
i_{1}
\end{array}\right)\left(\begin{array}{c}
K_{2}+i_{2}-1 \\
i_{2}
\end{array}\right) \cdots \\
& \cdot\left(\begin{array}{c}
K_{m}+i_{m}-1 \\
i_{m}
\end{array}\right) \alpha_{1}^{i_{1} \alpha_{2}^{i_{2}} \cdots \alpha_{m}^{i_{m}},} \\
& i_{1}+i_{2}+\cdots+i_{m}=r,
\end{aligned}
$$

where $K_{1}, K_{2}, \cdots, K_{m}$ are positive real numbers. Now we define

$$
\begin{gathered}
E_{r}\left(K_{1}, K_{2}, \cdots, K_{m}\right)=\frac{E_{r}^{1}\left(K_{1}, K_{2}, \cdots, K_{m}\right)}{\left(\begin{array}{c}
\left.K_{1}+K_{2}+\cdots+K_{m}\right) \\
r
\end{array}\right)} \\
H_{r}\left(K_{1}, K_{2}, \cdots, K_{m}\right)=\frac{H_{r}^{1}\left(K_{1}, K_{2}, \cdots, K_{m}\right)}{\left(\begin{array}{c}
K_{1}+K_{2}+\cdots+K_{m}+r-1 \\
r
\end{array}\right) ;}
\end{gathered}
$$

we note that 


$$
\begin{gathered}
E_{r}(K, K, K, \cdots, K)=E_{r}(K) \quad \text { and } \\
H_{r}(K, K, \cdots, K)=H_{r}(K), \\
E_{r}(1,1, \cdots, 1)=E_{r} \text { and } H_{r}(1,1, \cdots, 1)=H_{r} .
\end{gathered}
$$

The generating series for (1.13) is

$$
1+\sum E_{r}^{1}\left(K_{1}, K_{2}, \cdots, K_{m}\right) x^{r}=\prod_{i=1}^{m}\left(1+\alpha_{i} x\right)^{K_{i}}
$$

and the generating series for (1.14) is

$$
1+\sum H_{r}^{1}\left(K_{1}, K_{2}, \cdots, K_{m}\right) x^{r}=\prod_{i=1}^{m}\left(1-\alpha_{i} x\right)^{-K_{i}}
$$

where $K_{1}, K_{2}, \cdots, K_{m}$ are positive real numbers. In the proof of the theorem we make use of the classical theory of maxima and minima as in [1].

2. Lемма 1. If $r=1$, then for all $m$,

$$
\begin{aligned}
{\left[H _ { r } \left(K_{1}, K_{2}, \cdots\right.\right.} & \left.\left., K_{m}\right)\right]^{2} \\
& \leqq H_{r-1}\left(K_{1}, K_{2}, \cdots, K_{m}\right) H_{r+1}\left(K_{1}, K_{2}, \cdots, K_{m}\right)
\end{aligned}
$$

where $\alpha_{1}, \alpha_{2}, \cdots, \alpha_{m}$ are nonnegative reals. The inequality is strict unless all the variables are equal.

Proof.

$$
\begin{gathered}
H_{1}\left(K_{1}, K_{2}, \cdots, K_{m}\right)=\frac{\left(\sum_{i=1}^{m} K_{i} \alpha_{i}\right)}{\left(\sum_{i=1}^{m} K_{i}\right)} \\
H_{2}\left(K_{1}, K_{2}, \cdots, K_{m}\right)=\frac{\sum_{i=1}^{m} K_{i}\left(K_{i}+1\right) \alpha_{i}^{2}+\sum_{i \neq j} 2 K_{i} K_{j} \alpha_{i} \alpha_{j}}{\left(\sum_{i=1}^{m} K_{i}\right)\left(\sum_{i=1}^{m} K_{i}+1\right)} .
\end{gathered}
$$

Hence

$H_{2}\left(K_{1}, K_{2}, \cdots, K_{m}\right)-\left[H_{1}\left(K_{1}, K_{2}, \cdots, K_{m}\right)\right]^{2}$$$
=\frac{1}{\left(\Sigma_{i=1}^{m} K_{i}\right)^{2}\left(\Sigma_{i=1}^{m} K_{i}+1\right)}
$$$$
\cdot\left\{\left(\sum_{i=1}^{m} K_{i}\right)\left[\sum_{i=1}^{m} K_{i}\left(K_{i}+1\right) \alpha_{i}^{2}+2 \sum_{i \neq j} K_{i} K_{j} \alpha_{i} \alpha_{j}\right]\right.
$$$$
\left.-\left(\sum_{i=1}^{m} K_{i}+1\right)\left(\sum_{i=1}^{m} K_{i} \alpha_{i}\right)^{2}\right\} \text {. }
$$ 
Or,

$$
\begin{aligned}
H_{2}\left(K_{1}, K_{2}, \cdots, K_{m}\right)-\left[H_{1}\left(K_{1}, K_{2}, \cdots, K_{m}\right)\right]^{2} \\
\quad=\frac{1}{\left(\sum_{i=1}^{m} K_{i}\right)^{2}\left(\sum_{i=1}^{m} K_{i}+1\right)}\left\{\sum_{i \neq j} K_{i} K_{j}\left(\alpha_{i}-\alpha_{j}\right)^{2}\right\} .
\end{aligned}
$$

Hence the result.

3. Leмма 2. If $r=1$, then for all $m$,

$$
\begin{aligned}
{\left[E_{r}\left(K_{1}, K_{2}, \cdots, K_{m}\right)\right]^{2} } & \\
& \geqq E_{r-1}\left(K_{1}, K_{2}, \cdots, K_{m}\right) E_{r+1}\left(K_{1}, K_{2}, \cdots, K_{m}\right)
\end{aligned}
$$

where $\alpha_{1}, \alpha_{2}, \cdots, \alpha_{m}$ are nonnegative reals. The inequality is strict unless all the variables are equal.

Proof. Same as Lemma 2.

4. Lemma 3. For all $r$, if $m=1$, then

$$
\begin{aligned}
H_{r-1}\left(K_{1}, K_{2}, \cdots, K_{m}\right) H_{r+1}\left(K_{1}, K_{2}, \cdots, K_{m}\right) & \\
- & {\left[H_{r}\left(K_{1}, K_{2}, \cdots, K_{m}\right)\right]^{2}=0 }
\end{aligned}
$$

and

$$
\begin{aligned}
E_{r-1}\left(K_{1}, K_{2}, \cdots, K_{m}\right) E_{r+1}\left(K_{1},\right. & \left.K_{2}, \cdots, K_{m}\right) \\
& -\left[E_{r}\left(K_{1}, K_{2}, \cdots, K_{m}\right)\right]^{2}=0 .
\end{aligned}
$$

Proof. Follows from (1.15) and (1.16).

5. Lemma 4.

$$
\begin{aligned}
& \sum_{i=1}^{m} \frac{\partial}{\partial \alpha_{i}} H_{r}^{1}\left(K_{1}, K_{2}, \cdots, K_{m}\right) \\
& =\left(\sum_{i=1}^{m} K_{1}+r-1\right) H_{r-1}\left(K_{1}, K_{2}, \cdots, K_{m}\right) \text {, } \\
& \sum_{i=1}^{m} \frac{\partial}{\partial \alpha_{i}} E_{r}^{1}\left(K_{1}, K_{2}, \cdots, K_{m}\right) \\
& =\left(\sum_{i=1}^{m} K_{i}-r+1\right) E_{r-1}\left(K_{1}, K_{2}, \cdots, K_{m}\right) .
\end{aligned}
$$

Proof. By Euler's theorem on homogeneous functions we have

$$
\sum_{i=1}^{m} \alpha_{i} \frac{\partial}{\partial \alpha_{i}} H_{r}^{1}\left(K_{1}, K_{2}, \cdots, K_{m}\right)=r H_{r}^{1}\left(K_{1}, K_{2}, \cdots, K_{m}\right) .
$$


From (1.20) and (5.3) we get (5.1).

In the same way (5.2) can be proved from (1.19).

6. TheOREM 1. If $\alpha_{1}, \alpha_{2}, \cdots, \alpha_{m}$ are nonnegative reals, then

$$
\begin{aligned}
H_{r-1}\left(K_{1}, K_{2}, \cdots, K_{m}\right) H_{r+1}\left(K_{1}, K_{2}, \cdots, K_{m}\right) & \\
& >\left[H_{r}\left(K_{1}, K_{2}, \cdots, K_{m}\right)\right]^{2}
\end{aligned}
$$

and

$$
H_{r}\left(K_{1}, K_{2}, \cdots, K_{m}\right)^{1 / r}>H_{r+1}\left(K_{1}, K_{2}, \cdots, K_{m}\right)^{1 /(r+1)}
$$

where $r=1,2,3, \cdots$. The inequality is strict unless all the variables are equal.

Proof. Let $\alpha_{1}, \alpha_{2}, \cdots, \alpha_{m}$ be $m$ variables subject to the following conditions:

$$
\alpha_{1}>0, \quad \alpha_{2}>0, \cdots, \alpha_{m}>0
$$

and

$$
H_{r-1}^{1}\left(K_{1}, K_{2}, \cdots, K_{m}\right) H_{r+1}^{1}\left(K_{1}, K_{2}, \cdots, K_{m}\right)=1 .
$$

These conditions define a closed set of points in $m$-dimensional space. This set of points is bounded also since the function

$$
H_{r-1}^{1}\left(K_{1}, K_{2}, \cdots, K_{m}\right)
$$

and

$$
H_{r+1}^{1}\left(K_{1}, K_{2}, \cdots, K_{m}\right)
$$

has positive coefficients and includes the term $\alpha_{i}^{2 r}$ for $i=1,2, \cdots, m$. Also from Lemma 1 the theorem is true for all $m$ if $r=1$, and by Lemma 3 , the theorem is true for all $r$, if $m=1$. We use double induction on $m$ and $r$ to prove the theorem. We shall prove that it holds for all $m$ and $r$ provided it holds for all pairs $m, r$ with $m_{1}<m$, and for all pairs $m, r_{1}$ with $r_{1}<r$. By Euler's theorem on homogeneous functions and (6.3)

$$
\begin{aligned}
\sum \alpha_{i} & \frac{\partial}{\partial \alpha_{i}} H_{r-1}^{1}\left(K_{1}, K_{2}, \cdots, K_{m}\right) H_{r+1}^{1}\left(K_{1}, K_{2}, \cdots, K_{m}\right) \\
& =2 r H_{r-1}^{1}\left(K_{1}, K_{2}, \cdots, K_{m}\right) H_{r+1}^{1}\left(K_{1}, K_{2}, \cdots, K_{m}\right)=2 r .
\end{aligned}
$$

From (6.4) we note that the first partial derivatives cannot all vanish at the point at which $\left[H_{r}^{1}\left(K_{1}, K_{2}, \cdots, K_{m}\right)\right]^{2}$ takes its maximum value, say $M$. Applying Lagrange's conditions at this point we have 


$$
\begin{aligned}
\frac{\partial}{\partial \alpha_{i}} & {\left[H_{r}^{1}\left(K_{1}, K_{2}, \cdots, K_{m}\right)\right]^{2} } \\
& -\lambda \frac{\partial}{\partial \alpha_{i}} H_{r-1}^{1}\left(K_{1}, K_{2}, \cdots, K_{m}\right) H_{r+1}^{1}\left(K_{1}, K_{2}, \cdots, K_{m}\right)=0 .
\end{aligned}
$$

From (6.5) we have

$$
\begin{aligned}
2 H_{r}^{1}\left(K_{1}, K_{2}, \cdots, K_{m}\right) \frac{\partial}{\partial \alpha_{i}} H_{r}^{1}\left(K_{1}, K_{2}, \cdots, K_{m}\right) \\
=\lambda\left\{H_{r-1}^{1}\left(K_{1}, K_{2}, \cdots, K_{m}\right) \frac{\partial}{\partial \alpha_{i}} H_{r+1}^{1}\left(K_{1}, K_{2}, \cdots, K_{m}\right)\right. \\
\left.\quad+H_{r+1}^{1}\left(K_{1}, K_{2}, \cdots, K_{m}\right) \frac{\partial}{\partial \alpha_{i}} H_{r-1}^{1}\left(K_{1}, K_{2}, \cdots, K_{m}\right)\right\} .
\end{aligned}
$$

Now multiplying each of (6.6) by $\alpha_{i}$ and adding we have

$$
\begin{gathered}
2 H_{r}^{1}\left(K_{1}, K_{2}, \cdots, K_{m}\right) \sum_{i=1}^{m} \alpha_{i} \frac{\partial}{\partial \alpha_{i}} H_{r}^{1}\left(K_{1}, K_{2}, \cdots, K_{m}\right) \\
-\lambda\left\{H_{r-1}^{1}\left(K_{1}, K_{2}, \cdots, K_{m}\right) \sum_{i=1}^{m} \alpha_{i} \frac{\partial}{\partial \alpha_{i}} H_{r+1}^{1}\left(K_{1}, K_{2}, \cdots, K_{m}\right)\right. \\
\left.+H_{r+1}^{1}\left(K_{1}, K_{2}, \cdots, K_{m}\right) \sum_{i=1}^{m} \alpha_{i} \frac{\partial}{\partial \alpha_{i}} H_{r-1}^{1}\left(K_{1}, K_{2}, \cdots, K_{m}\right)\right\}=0 .
\end{gathered}
$$

But

$$
\begin{aligned}
\sum_{i=1}^{m} \alpha_{i} \frac{\partial}{\partial \alpha_{i}} H_{r+1}^{1}\left(K_{1}, K_{2}, \cdots, K_{m}\right) & \\
= & (r+1) H_{r+1}^{1}\left(K_{1}, K_{2}, \cdots, K_{m}\right) .
\end{aligned}
$$

Hence from (6.7) and (6.8) we have

$$
\begin{aligned}
& 2 r\left[H_{r}^{1}\left(K_{1}, K_{2}, \cdots, K_{m}\right)\right]^{2} \\
& \quad-\lambda 2 r H_{r-1}\left(K_{1}, K_{2}, \cdots, K_{m}\right) H_{r+1}\left(K_{1}, K_{2}, \cdots, K_{m}\right)=0
\end{aligned}
$$

or,

$$
\lambda=\frac{\left[H_{r}^{1}\left(K_{1}, K_{2}, \cdots, K_{m}\right)\right]^{2}}{\left[H_{r-1}^{1}\left(K_{1}, K_{2}, \cdots, K_{m}\right) H_{r+1}^{1}\left(K_{1}, K_{2}, \cdots, K_{m}\right)\right]} .
$$

Now using (6.6) 


$$
\begin{aligned}
& 2 H_{r}^{1}\left(K_{1}, K_{2}, \cdots, K_{m}\right)\left(\sum_{i=1}^{m} K_{i}+r-1\right) H_{r-1}^{1}\left(K_{1}, K_{2}, \cdots, K_{m}\right) \\
&=\lambda\left\{H_{r-1}^{1}\left(K_{1}, K_{2}, \cdots, K_{m}\right)\left(\sum_{i=1}^{m} K_{i}+r\right)\right. \\
& \cdot H_{r}^{1}\left(K_{1}, K_{2}, \cdot \cdots, K_{m}\right)+H_{r}^{1}\left(K_{1}, K_{2}, \cdots, K_{m}\right) \\
&\left.\cdot\left(\sum_{i=1}^{m} K_{i}+r-2\right) H_{r-2}^{1}\left(K_{1}, K_{2}, \cdots, K_{m}\right)\right\} .
\end{aligned}
$$

Now from (6.11) we have,

$$
\begin{aligned}
& 2\left\{\sum_{i=1}^{m} K_{i}+r-1\right\}-\lambda\left\{\sum_{i=1}^{m} K_{i}+r\right\} \\
&=\lambda\left\{\sum_{i=1}^{m} K_{i}+r-2\right\} \\
& \cdot \frac{H_{r+1}^{1}\left(K_{1}, K_{2}, \cdots, K_{m}\right) H_{r-2}^{1}\left(K_{1}, K_{2}, \cdots, K_{m}\right)}{H_{r}^{1}\left(K_{1}, K_{2}, \cdots, K_{m}\right) H_{r-1}^{1}\left(K_{1}, K_{2}, \cdots, K_{m}\right)} .
\end{aligned}
$$

Now from (6.12) and (6.10) we have

$$
\begin{aligned}
& 2\left\{\sum_{i=1}^{m} K_{i}+r-1\right\}-\lambda\left\{\sum_{i=1}^{m} K_{i}+r\right\} \\
&=\left\{\sum_{i=1}^{m} K_{i}+r-2\right\} \\
& \cdot \frac{H_{r}^{1}\left(K_{1}, K_{2}, \cdots, K_{m}\right) H_{r-2}^{1}\left(K_{1}, K_{2}, \cdots, K_{m}\right)}{\left[H_{r-1}^{1}\left(K_{1}, K_{2}, \cdots, K_{m}\right)\right]^{2}} .
\end{aligned}
$$

But by induction hypothesis

$$
\begin{array}{r}
\frac{H_{r}^{1}\left(K_{1}, K_{2}, \cdots, K_{m}\right) H_{r-2}^{1}\left(K_{1}, K_{2}, \cdots, K_{m}\right)}{\left[H_{r-1}\left(K_{1}, K_{2}, \cdots, K_{m}\right)\right]^{2}} \\
\geqq \frac{\left(\sum_{i=1}^{m} K_{i}+r-1\right)}{\left(\sum_{i=1}^{m} K_{i}+r-2\right)} \frac{(r-1)}{r} .
\end{array}
$$

Hence 


$$
\begin{aligned}
2\left(\sum_{i=1}^{m} K_{i}+r-1\right)- & \lambda\left(\sum_{i=1}^{m} K_{i}+r\right) \\
& \geqq\left(\sum_{i=1}^{m} K_{i}+r-2\right) \frac{r-1}{r} \frac{\left(\sum_{i=1}^{m} K_{i}+r-1\right)}{\left(\sum_{i=1}^{m} K_{i}+r-2\right)} .
\end{aligned}
$$

Therefore,

$$
2\left(\sum_{i=1}^{m} K_{i}+r-1\right)-\lambda\left(\sum_{i=1}^{m} K_{i}+r\right) \geqq \frac{r-1}{r}\left(\sum_{i=1}^{m} K_{i}+r-1\right)
$$

or

$$
\left(\sum_{1}^{m} K_{i}+r-1\right) \frac{r+1}{r} \geqq \lambda\left(\sum_{i=1}^{m} K_{i}+r\right)
$$

or

$$
\lambda<\frac{r+1}{r} \frac{\left(\sum_{i=1}^{m} K_{i}+r-1\right)}{\left(\sum_{i=1}^{m} K_{i}+r\right)} .
$$

In the next place we suppose that the maximum $M$ is attained when $\alpha_{m_{1+1}}=0, \alpha_{m_{1+2}}=0, \cdots, \alpha_{m}=0, \alpha_{1} \neq 0, \cdots, \alpha_{m_{1}} \neq 0,\left(m_{1}<m\right)$. Induction on $m$ gives

$$
\lambda=\frac{\left[H_{r}^{1}\left(K_{1}, K_{2}, \cdots, K_{m_{1}}\right)\right]^{2}}{H_{r-1}^{1}\left(K_{1}, K_{2}, \cdots, K_{m_{1}}\right) H_{r+1}^{1}\left(K_{1}, K_{2}, \cdots, K_{m_{1}}\right)} ;
$$

then

$$
\lambda \leqq \frac{r+1}{r} \frac{\left(\sum_{i=1}^{m_{1}} K_{i}+r-1\right)}{\left(\sum_{i=1}^{m_{1}} K_{i}+r\right)},
$$

and clearly

$$
\frac{r+1}{r} \frac{\sum_{i=1}^{m_{1}} K_{i}+r-1}{\sum_{i=1}^{m_{1}} K_{i}+r}<\frac{r+1}{r} \frac{\sum_{i=1}^{m} K_{i}+r-1}{\sum_{i=1}^{m} K_{i}+r} ;
$$

hence the theorem.

The second part of the theorem can be proved as in [2].

7. ThEOREM 2. If $\alpha_{1}, \alpha_{2}, \cdots, \alpha_{m}$ are nonnegative reals, then

$$
\begin{aligned}
{\left[E_{r}\left(K_{1}, K_{2}, \cdots, K_{m}\right)\right]^{2} } & \\
& >E_{r-1}\left(K_{1}, K_{2}, \cdots, K_{m}\right) E_{r+1}\left(K_{1}, K_{2}, \cdots, K_{m}\right)
\end{aligned}
$$


and

$$
E_{r}\left(K_{1}, K_{2}, \cdots, K_{m}\right)^{1 / r}>E_{r}\left(K_{1}, K_{2}, \cdots, K_{m}\right)^{1 / r+1},
$$

where $r=1,2, \cdots, r<K+1$, where $K$ is not integral and $K$ denotes the smallest of the real positive numbers, $K_{1}, K_{2}, \cdots, K_{m}$. The inequality is strict unless all the variables are equal.

Proof. The restriction on $r$, makes all the terms positive, and hence the theorem can be proved in the same way as Theorem 1 .

\section{REFERENCES}

1. J. N. Whiteley, A generalization of a theorem of Newton, Proc. Amer. Math. Soc. 13 (1962), 144-151.

2. G. H. Hardy, J. E. Littlewood and G. P6lya, Inequalities, Cambridge Univ. Press, Cambridge, 1952, p. 52.

DALHOUSIE UNIVERSITY 